\title{
Timing of antenatal care and ART initiation in HIV-infected pregnant women before and after introduction of NIMART
}

\author{
C N Mnyani, ${ }^{1,2}$ MB ChB, FCOG; E Marinda, ${ }^{3,4} \mathrm{PhD} ;$ H Struthers, ${ }^{2,5} \mathrm{MSc}, \mathrm{MBA} ; \mathbf{M}$ Gulley; ${ }^{2}$ R Machepa $;{ }^{2}$ \\ J McIntyre, ${ }^{2,6} \mathrm{MB} \mathrm{ChB}$, FRCOG \\ ${ }^{1}$ Department of Obstetrics and Gynaecology and School of Public Health, Faculty of Health Sciences, University of the Witwatersrand, \\ Johannesburg, South Africa \\ ${ }^{2}$ Anova Health Institute, Johannesburg, South Africa \\ ${ }^{3}$ Health and Development Africa, Mott MacDonald South Africa \\ ${ }^{4}$ Epidemiology and Biostatistics Unit, School of Public Health, Faculty of Health Sciences, University of the Witwatersrand, \\ Johannesburg, South Africa \\ ${ }^{5}$ Department of Infectious Diseases, Faculty of Health Sciences, University of Cape Town, South Africa \\ ${ }^{6}$ School of Public Health and Family Medicine, University of Cape Town, South Africa
}

Corresponding author: C N Mnyani (coceka.mnyani@wits.ac.za)

\begin{abstract}
In this review of routinely collected data from five community health centres in the Johannesburg Health District, we assess timing of antenatal care and antiretroviral therapy (ART) initiation in HIV-infected pregnant women before and after the introduction of nurse-initiated management of ART in antenatal clinics. There are important lessons to be learnt as we reflect on the South African prevention of mother-to-child transmission of HIV programme.
\end{abstract}

S Afr J HIV Med 2014;15(2):55-56. DOI:10.7196/SAJHIVMED.1009

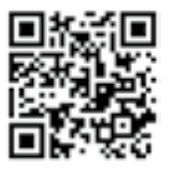

It is widely acknowledged that the highest risk of mother-to-child transmission (MTCT) of HIV is in HIV-infected women with low $\mathrm{CD} 4^{+}$counts, who are eligible for antiretroviral therapy (ART) ${ }^{[1]}$ Timely initiation of ART in this group is critical to decreasing paediatric HIV infection and HIV-related maternal morbidity and mortality. ${ }^{[2,3]}$ ART initiation in pregnancy is also associated with better maternal immunological and virological outcomes compared with starting ART after pregnancy. ${ }^{[4]}$

Prior to the current prevention of MTCT (PMTCT) guidelines, criteria for ART initiation in pregnant women were based on a CD4 $4^{+}$count of $\leq 350$ cells $/ \mu$ l, or World Health Organization (WHO) stage 3 or 4 disease regardless of $\mathrm{CD} 4^{+}$ count. ${ }^{[5]}$ Reflecting on the history of the PMTCT programme in South Africa, two of the main early challenges to initiating ART in pregnancy were that HIV treatment sites were physically separate from antenatal clinics and ART initiation was largely physician led. ${ }^{[6]}$ This led to delays in referral and initiating treatment, and as a result, a significant proportion of ARTeligible pregnant women would go through pregnancy without starting treatment. ${ }^{[7]}$ Nurse-initiated and managed ART (NIMART) in antenatal clinics was introduced to address these challenges, supported by evidence that integration of ART with antenatal care decreases time to initiation and increases the proportion of pregnant women initiated..$^{[8-10]}$ Pregnant women need to access antenatal care early for timeous treatment initiation.
This retrospective review of routinely collected data assesses timing of antenatal care and ART initiation in HIV-infected, eligible pregnant women presenting to five community health centres in the Johannesburg Health District.

\section{Method}

Time to initiation in pregnant women who presented prior to the introduction of NIMART was compared with those who presented after. Between October 2010 and March 2012, a total of 1436 ART-eligible pregnant women were identified.

The study was approved by the University of Cape Town's Human Research Ethics Committee, and by the Gauteng Province office for policy, planning and research.

\section{Results}

Characteristics of the women are presented in Table 1. The mean gestational age when accessing antenatal care was 19.2 weeks (standard deviation (SD) of 6.6), and the mean gestational age at ART initiation was 24.6 weeks (SD 6.2). There was no significant reduction in time to initiation after the introduction of NIMART. Overall, the median time to initiation prior to the introduction of NIMART was 3.4 weeks (interquartile range (IQR) 2.0 - 5.9) whereas after the introduction of NIMART, it was 3.0 weeks (IQR 1.4 - 5.4). Assessing data from individual clinics, there was evidence of an increase in time to starting ART in some facilities. However, overall there was an increase in the proportion of eligible pregnant women who started ART after the introduction of NIMART, in all the facilities. Data on pregnancy outcomes were 
Table 1. Baseline characteristics and timing of ART initiation

\begin{tabular}{|c|c|c|c|c|c|}
\hline & \multicolumn{5}{|c|}{ Facilities } \\
\hline & $\mathbf{A}$ & B & C & $\mathbf{D}$ & $\mathbf{E}$ \\
\hline \multicolumn{6}{|c|}{ Baseline CD4+ count (cells/ $\mu \mathrm{l})$} \\
\hline Mean $( \pm S D)$ & $211( \pm 85)$ & $223( \pm 86)$ & $215( \pm 87)$ & $240( \pm 112)$ & $228( \pm 81)$ \\
\hline Median (IQR) & $214(151-284)$ & $234(147-299)$ & $225(152-286)$ & $245(174-299)$ & $235(169-296)$ \\
\hline Range & $15-344$ & $27-350$ & $23-350$ & $25-650$ & $45-351$ \\
\hline \multicolumn{6}{|c|}{ Gestational age at 1st antenatal visit (weeks) } \\
\hline Mean $( \pm \mathrm{SD})$ & $20.7( \pm 6.4)$ & $17.4( \pm 6.2)$ & $19.9( \pm 5.9)$ & $18.1( \pm 6.9)$ & $19.5( \pm 6.9)$ \\
\hline Median (IQR) & $20(16-24)$ & $18(12-20)$ & $20(16-24)$ & $16(12-24)$ & $20(15-24)$ \\
\hline Range & $8-36$ & $4-30$ & $8-32$ & $4-36$ & $5-36$ \\
\hline \multicolumn{6}{|c|}{ Gestational age at ART initiation (weeks) } \\
\hline Mean $( \pm S D)$ & $25.4( \pm 6.4)$ & $22.0( \pm 5.8)$ & $24.3( \pm 5.5)$ & $24.6( \pm 6.6)$ & $25.1( \pm 6.3)$ \\
\hline Median (IQR) & $25(21-30)$ & $21(18-27)$ & $25(20-28)$ & $24(20-30)$ & $25(21-30)$ \\
\hline Range & $12-37$ & $8-34$ & $14-36$ & $11-39$ & $10-38$ \\
\hline \multicolumn{6}{|c|}{$\begin{array}{l}\text { Time to initiation prior to NIMART } \\
\text { (weeks) }\end{array}$} \\
\hline Mean $( \pm \mathrm{SD})$ & $1.6( \pm 0.5)$ & $4.0( \pm 2.7)$ & $5.5( \pm 2.5)$ & $2.6( \pm 0.8)$ & $3.3( \pm 1.8)$ \\
\hline Median (IQR) & $1.9(1.3-2.3)$ & $3.1(2.0-5.1)$ & $5.6(1.7-7.0)$ & $2.4(1.9-3.2)$ & $2.8(1.9-5.1)$ \\
\hline Range & $1.0-2.3$ & $0.9-10.6$ & $1.1-12.6$ & $1.6-4.0$ & $1.3-5.9$ \\
\hline \multicolumn{6}{|c|}{ Time to initiation after NIMART (weeks) } \\
\hline Mean $( \pm S D)$ & $2.0( \pm 1.9)$ & $4.4( \pm 3.3)$ & $3.8( \pm 3.5)$ & $5.7( \pm 3.8)$ & $6.6( \pm 3.9)$ \\
\hline Median (IQR) & $1.3(1.0-2.3)$ & $3.7(2.4-5.1)$ & $2.1(1.4-4.9)$ & $4.1(3.0-8.1)$ & $6.0(4.0-8.8)$ \\
\hline Range & $0-9.7$ & $0-18.9$ & $0.7-15.7$ & $0.7-18.4$ & $0.6-18.7$ \\
\hline
\end{tabular}

available for $61.8 \%$ of the women, namely 881 live births, 1 neonatal death, 4 stillbirths and 2 miscarriages. Of the 881 infants tested at $\sim 6$ weeks of age, only two $(0.2 \%)$ were HIV-infected.

\section{Discussion}

The data provided an interesting reflection on the PMTCT programme. While pregnant women in the review accessed antenatal care relatively late, in the second trimester, there were no lengthy delays in initiating ART in patients who reached the HIV management sites, even prior to the introduction of NIMART in antenatal clinics. Despite several challenges in the antiretroviral (ARV) roll-out in the past few years, the data showed that there were pockets of excellence. In the facilities where the intervention increased time to initiation, there are several possible reasons for this: there could have been a shortage of skilled and properly trained staff to manage ART-eligible pregnant women in the antenatal clinics; and during the early stages of the introduction of NIMART, antenatal clinics were only able to initiate treatment on select days.

\section{Conclusion}

While the updated PMTCT guidelines recommend starting all HIVinfected pregnant women on ART at the first antenatal visit, to reduce delays in initiating therapy, focus needs to shift from quantity to quality. As the number of patients on treatment increases, clinicians need to ensure that they provide high-quality services with appropriate clinical and laboratory monitoring, and long-term retention of patients in care.

\section{References}

1. McIntyre J. Use of antiretrovirals during pregnancy and breastfeeding in low-income and middle-income countries. Curr Opin HIV AIDS 2010;5(1):48-53. [http://dx.doi. org/10.101097/COH.0b013e328333b8ab]

2. Sturt AS, Dokubo EK, Sint TT. Antiretroviral therapy (ART) for treating HIV infection in ART-eligible pregnant women. Cochrane Database Syst Rev 2010;17(3):CD008440.

3. Marazzi MC, Palombi L, Nielsen-Saines K, et al. Extended antenatal use of triple antiretroviral therapy for prevention of mother-to-child transmission of HIV-1 correlates with favourable pregnancy outcomes. AIDS 2011;25(13):1611-1618. [http:// dx.doi.org/10.1097/QAD.0b013e3283493ed0]

4. Melekhin VV, Shepherd BE, Stinnette SE, et al. Antiretroviral therapy initiation before, during, or after pregnancy in HIV-1-infected women: Maternal virologic, immunologic, and clinical response. PLoS ONE 2009;4(9):e6961. [http://dx.doi.org/10.1371/journal. pone.0006961]

5. National Department of Health, South Africa; South African National AIDS Council. Clinical Guidelines: PMTCT (Prevention of Mother-to-Child Transmission), 2010. Pretoria: South African National Department of Health, 2010.

6. Dohrn J, Nzama B, Murrman M. The impact of HIV scale-up on the role of nurses in South Africa: Time for a new approach. J Acquir Immune Defic Syndr 2009;52:S27-S29. [http://dx.doi.org/10.1097/QAI.0b013e3181bbc9e4]

7. Myer L. Initiating antiretroviral therapy in pregnancy: The importance of timing. J Acquir Immune Defic Syndr 2011;58(2):125-126. [http://dx.doi.org/10.1097/ QAI.0b013e31822ad573]

8. Van der Merwe K, Chersich MF, Technau K, Umurungi Y, Conradie F, Coovadia A. Integration of antiretroviral treatment within antenatal care in Gauteng Province, South Africa. J Acquir Immune Defic Syndr 2006;43(5):577-581. [http://dx.doi. org/10.1097/01.qai.0000243099.72770.d2]

9. Killam WP, Tambatamba BC, Chintu N, et al. Antiretroviral therapy in antenatal care to increase treatment initiation in HIV-infected pregnant women: A stepped-wedge evaluation. AIDS 2010;24(1):85-91. [http://dx.doi.org/10.1097/ QAD.0b013e32833298be]

10. Stinson K, Jennings K, Myer L. Integration of antiretroviral therapy services into antenatal care increases treatment initiation during pregnancy: A cohort study. PLoS ONE 2013;8(5):e63328. [http://dx.doi:10.1371/journal.pone.0063328] 